\title{
Stem cell therapy in the treatment of inflammatory bowel disease
}

\begin{abstract}
The application of cell therapy in the treatment of inflammatory bowel disease currently is of increasing scientific interest. Cell therapy in the treatment of Crohn's disease and ulcerative colitis includes 2 approaches: immunosuppressant followed by hematopoietic stem cells transplantation and systemic or local application of mesenchymal stem cells. The latter were reported to exhibit immunosuppressive and regenerative effect. Actually more than 1,500 patients with inflammatory bowel disease have been treated with mesenchymal stem cells worldwide. The introduction of cell therapy into gastroenterology will significantly expand the arsenal of therapeutic arrangements for the treatment of inflammatory bowel disease, in particular resistant forms of the disease and will allow to increase the effectiveness of treatment.
\end{abstract}

Keywords: crohn's disease, ulcerative colitis, stem cells
Volume I Issue I - 2014

Nasadyuk CM
Danylo Halytsky Lviv National Medical University, Ukraine

Correspondence: Christina Nasadyuk, Danylo Halytsky Lviv National Medical University, 69, Pekarska Street, 79000 Lviv, Ukraine,Tel +38-98-409-1958, Email nasadyukch@gmail.com

Received: April 22, 2014 | Published: May 21, 2014
Abbreviations: IBD, inflammatory bowel disease; UC, ulcerative colitis; CD, crohn's diseases; TNF- $\alpha$, tumor necrosis factor $\alpha$; IL-12/23, interleukin 12/23; IL-6R, interleukin 6R; iRNA, information ribonucleic acid; SC, stem cells; CT, cell therapy; MSC, mesenchymal stem cells; HSC, hematopoietic stem cells; 2,4,6TNBA, 2,4,6-trinitrobenzene sulfonic acid; SDF-1, stromal derived factor-1; VEGF, vasculoendothelial growth factor; EGF, epidermal growth factor; DSS, dextran sulphate sodium; HSCT, hematopoietic stem cells transplantation; EPC, endothelial progenitor cells

\section{Introduction}

Achievements of modern science changed the ideas about etiology and pathogenesis of IBD, mainly $\mathrm{UC}$ and $\mathrm{CD}$, what resulted in the emergence of novel approaches to the treatment of these disorders. ${ }^{1-6}$ Environmental, genetic factors and microbial flora were elicited to play an important role in the development of inflammatory process in the intestinal wall, activating immune response. ${ }^{1,7,8}$ The disruption of balance between proinflammatory and anti-inflammatory factors in intestinal mucosa becomes the trigger of the development of pathologic process in which numerous cellular and molecular mechanisms are envolved. ${ }^{1,7}$ Genetic nature of IBD is confirmed by wide studies of human genome and studies on twins. ${ }^{3}$ The efficiency of conventional methods of UC and CD treatment leaves much to be desired. ${ }^{1,9-11}$ Modern therapy of IBD includes biological therapy (antibodies to TNF- $\alpha$, adhesion molecules, IL-12/23, IL-6R, endoscopic application of artificially grown pieces of tissues etc) and gene therapy (iRNAtechnologies aimed at inhibition of the necessary genes). . $, 3,7,12,13^{\text {The }}$ application of nanoparticles for the transportation of drugs despite its high cost is also promising. ${ }^{2}$

In recent years an increasing number of experimental and clinical studies are devoted to the evaluation of therapeutic potential and effectiveness of SC in the treatment of UC and CD..$^{7,-11,14,15}$ According to experts' prognoses, CT will become the next generation of therapeutic arrangements in the treatment of IBD, in particular for patients with the secondary resistance to anticytokine therapy. ${ }^{4,12}$

\section{Discussion}

Actually several tens of adult SC is distinguished, that in health contribute to natural processes of tissues regeneration, play crucial role in wounds healing. ${ }^{16-18} \mathrm{SC}$ derived from bone marrow, peripheral and cord bloods, tissue of umbilical cord, amniotic fluid, adipose tissue have got wide use. , $16,17,19$ Remedial effect of SC is explained both by paracrine effect- stimulation of regenerative processes through the synthesis of growth factors and other biologically active compounds and ability to transformation into damaged tissues cells. $14,17,18,20,21$ Effectiveness of MSC in the treatment of autoimmune diseases is mediated by their potent immunosuppressive effect. ${ }^{22-24}$

Immunosuppressant followed by transplantation of alogeneic Hematopoietic Stem Cells (HSC) is a promising method of radical treatment of many autoimmune diseases, including $\mathrm{CD}$ and UC. ${ }^{5,11,23-}$ ${ }^{25}$ Another direction of CT of IBD is the use of MSC due to their immunosuppressant effect, what was mentioned above. ${ }^{9,10,23,24}$ In vivo and in vitro studies showed the interaction of MSC with regulatory T-lymphocytes ${ }^{22}$ In vitro studies also showed that MSC inhibit proliferation and cytotoxicity of T-lymphocytes, in particular natural killer cells, dendritic cells, production of cytokines, antibodies and antigen presentation what provides basis for the use of MSC in the treatment of autoimmune pathology. ${ }^{23,24}$

The decrease of clinical and histopathological signs of 2,4,6-TNBAinduced colitis, treated with MSC was accompanied by redistribution of regulatory T-lymphocytes from tissues to circulation..$^{22}$ SDF-1, produced by MSC was reported to play important role in chemo taxis regulation and $\mathrm{SC}$ engraftment in tissues, interacting with specific chemokine receptors. ${ }^{20}$

Another mechanism of therapeutic effect of MSC is their prominent regenerative effect as these cells are the precursors of the connective tissue. ${ }^{14,15,19}$ MSC were revealed to release numerous trophic factors (VEGF, EGF etc) and affect all stages of the complex process of wound healing - inflammatory, proliferative and tissue remodeling. ${ }^{21}$ The advantage of MSC use is the lack of immunogenicity what allows wider alogeneic application. ${ }^{14} \mathrm{MSC}$ above all are recommended to patients with fistulizing CD. ${ }^{14}$ Systemic MSC therapy was shown to be effective in resistant radiation colitis, decreasing pain, diarrhea, inflammation, bleeding, increasing the number of T-regulatory lymphocytes and decreasing of activated effector cells. ${ }^{14}$ 
Preclinical studies on the models of necrotizing colitis as well as experimental colitis induced by DSS and 2,4,6-TNBA showed positive effect of systemic and local use of MSC derived from the bone marrow, amniotic fluid, cord blood, adipose tissue on the course of colitis and stimulation of reparation. ${ }^{15,16,19,26} \mathrm{CT}$ was shown to increase survival and stimulate reparation of the damaged mucous membranes of the large intestine of newborn rats with necrotizing colitis. Animals were divided into 2 groups: control group was intraperitoneally injected bone marrow MSC and investigation group was treated with amniotic fluid SC. Positive effect of CT was accompanied by modulation of cyclooxygenase-2 expression in stromal cells of lamina propria of colonic mucosa. In amniotic fluid SC differentiated expression of genes of Wnt/beta-kinine pathway was detected as well as the migration of these cells and production of growth factors contributing to regeneration of colonic mucosa. ${ }^{15}$

In mice with DSS-induced colitis intraperitoneal injection of bone marrow or cord blood MSC was shown to decrease inflammation in colonic mucosa. Simultaneously the regulatory impact of MSC on the course of immune processes in spleen and mesenterial lymphatic nodes was noted. ${ }^{27}$ The application of adipose tissue MSC mixed with thrombin and fibrin significantly facilitated reparative processes in walls of the colon due to angiogenesis stimulation, what was proved in ischemia-induced colitis in mice. ${ }^{19}$ Immunosuppressive effects of MSC in the treatment of experimental colitis in mice were reported to be potentiated by interferon- $\gamma{ }^{26}$

Currently more than 200 clinical trials on MSC are registered worldwide, from which 22 trials are devoted to the treatment of autoimmune diseases and 27 - IBD. ${ }^{14}$ Due to literary data 1500 patients with IBD were carried out systemic and local treatment with MSC worldwide. The 1st and 2nd phases of clinical studies confirmed the safety of CT in the treatment of IBD and its advantages above the fibrin glue in fistulizing colitis., ${ }^{4}$

As mentioned above, CT is of special importance for the treatment of Patients with the resistance to anticytokine drugs. The level of anticytokine drugs antibodies was shown to correlate with the severity of clinical and endoscopic picture. ${ }^{12} \mathrm{CT}$ was also reported to decrease the need for glucocorticosteroid therapy and other drugs, what results in the decrease of treatment cost. ${ }^{28}$

MSC therapy was carried out in a group of 39 patients with UC and 11- with CD. Control groups included 20 patients with $\mathrm{UC}$ and 11 with $\mathrm{CD}$. Treatment with immunodepressing drugs was stopped 2-3 days before MSC introduction. Glucorticosteroids dose was decreased up to $15-20 \mathrm{mg} /$ day and aminosalicylates up to $2 \mathrm{~g} /$ day. After MSC transplantation significant decrease of inflammation signs was noted in 39 patients with UC and 11 patients with CD compared to control groups. In 40 patients clinical and morphological remission was stated. MSC therapy was ineffective in 8 patients with UC and 2 patients with $\mathrm{CD}$. In 34 from 50 patients with hormone-dependent or hormone-resistant forms of UC and CD MSC use allowed to decrease prednisolone dose up to $5 \mathrm{mg}$ /day. Thus, the authors consider MSC therapy as a novel strategic direction in the treatment of IBD due to its potent immunomodulating effect, decrease of autoimmune inflammation and stimulation of reparation processes in mucous membranes. ${ }^{9}$

Results of clinical studies give evidence that alogeneic MSC transplantation significantly increases remission duration, decreases the risk of relapse and efficiency is comparable with TNF $\alpha$ antibodies. In majority of patients MSC treatment allowed to discontinue 5-acetylsalicylic acid administration. Positive effect of CT was noted in hormone-resistant and hormone-dependent IBD. ${ }^{10}$ Another study reports about 3 patients with the active form of $\mathrm{CD}$, resistant to conventional therapy with the presence of antibodies to anti-TNF $\alpha$ drugs. These patients either refused from surgery or such treatment was not an acceptable alternative. 2 patients were successfully transplanted hematopoietic stem cells (HSC) with CD34+ selection. At the moment of examination 5 and 6 years after transplantation the patients were in remission. 1 patient got into remission after conditioning so HSCT was not performed. ${ }^{11}$

Effectiveness of SC was also noted in genetically mediated diseases of the intestine. HSCT was shown to restore gluten tolerability in patients with celiac disease. 2 clinical cases of successful HSCT after myeloablation in celiac disease were reported. After this treatment introduction of gluten-containing diet did not cause any clinical, serological or histological markers of the disease during 5 year follow-up period. ${ }^{5}$

Inherited deficiency of IL-10 and its receptors cause immune dysregulation in patients with the development of severe colitis, resistant to standard methods of treatment. HSCT was reported to be effective in patients with colitis on the background of deficiency of IL-10. ${ }^{25}$ Recent research data also gives evidence about the decrease of EPC number in patients with UC. Although, actually there is no data on the impact of infusion of EPC on the processes of mucosa regeneration. ${ }^{6}$

\section{Conclusion}

Introduction of $\mathrm{CT}$ into gastroenterology significantly expands the arsenal of therapeutic arrangements for IBD treatment, in particular forms of the disease resistant to conventional therapy. Results of longterm observations and improvement of protocols of SC application in patients with $\mathrm{CD}$ and $\mathrm{UC}$ will allow increasing the effectiveness of IBD treatment.

\section{Acknowledgements}

None.

\section{Funding}

None.

\section{Conflicts of Interest}

The author declares that there is no conflicts of interest.

\section{References}

1. Ricart E, Jauregui-Amezaga A, Ordas I, et al. Cell therapies for IBD: what works? Curr Drug Targets. 2013;14(12): 1453-1459.

2. Dhama K, Chakraborty S, Mahima, et al. Novel and emerging therapies safeguarding health of humans and their companion animals: a review. Pak J Biol Sci. 2013;16(3):101-111.

3. Parfenov AI. Crohn's disease: on the occasion of the 80th anniversary of description. Ter Arkh. 2013;85(8):35-42.

4. Van Deen WK, Oikonomopoulos A, Hommes DW. Stem cell therapy in inflammatory bowel disease: which, when and how? Curr Opin Gastroenterol. 2013;29(4):384-390.

5. Ciccocioppo R, Bernardo ME, Russo ML, et al. Allogeneic hematopoietic stem cell transplantation may restore gluten tolerance in patients with celiac disease. $J$ Pediatr Gastroenterol Nutr 2013;56(4):422-427. 
6. Deng X, Szabo S, Chen L, et al. New cell therapy using bone marrow-derived stem cells/endothelial progenitor cells to accelerate neovascularization in healing of experimental ulcerative colitis. Curr Pharm Des. 2011;17(16):1643-1651.

7. Fiorino G, Cesarini M, Danese S. Biological therapy for ulcerative colitis: what is after anti-TNF? Curr Drug Targets. 2011;12(10): 14331439.

8. Mokry M, Middendorp S, Wiegerinck CL, et al. Many inflammatory bowel disease risk Loci include regions that regulate gene expression in immune cells and the intestinal epithelium. Gastroenterology. 2014;146(4):1040-1047.

9. Lazebnik LB, Kniazev OV, Parfenov AI, et al. Transplantation of allogeneic mesenchymal stem cells from the bone marrow increases duration of remission and reduces the risk of ulcerative colitis relapse. Eksp Klin Gastroenterol. 2010;(3):5-10.

10. Parfenov AI. Inflammatory bowel diseases in the XXI century. Eksp Klin Gastroenterol. 2012;(3):3-6.

11. Hommes DW, Duijvestein M, Zelinkova Z, et al. Long-term followup of autologous hematopoietic stem cell transplantation for severe refractory Crohn's disease. J Crohns Colitis. 2011;5(6):543-549.

12. Kniazev OV, Parfenov AI, Ruchkina IN, et al. New possibilities for overcoming the secondary inefficiency of anti-cytokine therapy in patients with inflammatory bowel diseases. Ter Arkh. 2013;85(2):57-60.

13. Leggett CL, Gorospe EC, Lutzke L, et al. A new era: endoscopic tissue transplantation. Curr Opin Gastroenterol. 2013;29(5):495-500.

14. Voswinkel J, Francois S, Gorin NC, et al. Gastro-intestinal autoimmunity: preclinical experiences and successful therapy of fistulizing bowel diseases and gut graft versus host disease by mesenchymal stromal cells. Immunol Res. 2013;56(2-3):241-248.

15. Zani A, Cananzi M, Fascetti-Leon F, et al. Amniotic fluid stem cells improve survival and enhance repair of damaged intestine in necrotising enterocolitis via a COX-2 dependent mechanism. Gut. 2014;63(2):300-309.

16. Nasadyuk CM. The umbilical cord blood: medical and biological characteristics, storage, clinical use. Woman's Doctor. 2010;5:53-56.

17. Nasadyuk CM. Current status and perspectives of cord blood cry storage and clinical application in Ukraine. CTTE. 2011;6 (1):98-104.
18. Nasadyuk CM . Cell technologies in reproductology, obstetrics and gynaecology. Cell and organ transpl. 2013;1:61-65.

19. Joo HH, Jo HJ, Jung TD, et al. Adipose-derived stem cells on the healing of ischemic colitis: a therapeutic effect by angiogenesis. Int J Colorectal Dis. 2012;27(11):1437-1443.

20. Liu X, Zuo D, Fan H, et al. Over-expression of CXCR4 on mesenchymal stem cells protect against experimental colitis via immunomodulatory functions in impaired tissue. $J$ Mol Histol. 2014;45(2):181-193.

21. Watanabe S, Arimura Y, Nagaishi K, et al. Conditioned mesenchymal stem cells produce pleiotropic gut trophic factors. $J$ Gastroenterol. 2014;49(2):270-282.

22. Zuo D, Liu X, Shou Z, et al. Study on the interactions between transplanted bone marrow-derived mesenchymal stem cells and regulatory $\mathrm{T}$ cells for the treatment of experimental colitis. Int $\mathrm{J} \mathrm{Mol}$ Med. 2013;32(6):1337-1344.

23. De Miguel MP, Fuentes-Julian S, Blazquez-Martinez A, et al. Immunosuppressive properties of mesenchymal stem cells: advances and applications. Curr Mol Med. 2012;12(5):574-591.

24. Hayashi Y, Tsuji S, Tsujii M, et al. Topical implantation of mesenchymal stem cells has beneficial effects on healing of experimental colitis in rats. J Pharmacol Exp Ther. 2008;326(2):523-531.

25. Engelhardt KR, Shah N, Faizura-Yeop I, et al. Clinical outcome in IL10 - and IL-10 receptor-deficient patients with or without hematopoietic stem cell transplantation. J Allergy Clin Immunol. 2013;131(3):825-830.

26. Duijvestein M, Wildenberg ME, Welling MM, et al. Pretreatment with interferon $-\gamma$ enhances the therapeutic activity of mesenchymal stromal cells in animal models of colitis. Stem Cells. 2011;29(10):15491558 .

27. Li L, Liu S, Xu Y, et al. Human umbilical cord-derived mesenchymal stem cells down regulate inflammatory responses by shifting the Treg/Th17 profile in experimental colitis. Pharmacology. 2013;92(5-6):257-264.

28. Lazebnik LB, Gusein-Zade MG, Kniazev OV, et al. Pharmacoeconomic benefits of patients with ulcerative colitis treatment with help of mesenchymalstem cells. Eksp Klin Gastroenterol. 2010;(3):51-59. 